\title{
Immature Retinal Vasculature in Zone 2
}

National Cancer Institute

\section{Source}

National Cancer Institute. Immature Retinal Vasculature in Zone 2. NCI Thesaurus. Code C99118.

A finding that indicates the presence of immature retinal vessels in zone 2 of the retina. 